\title{
Aporte de Nitrógeno al suelo por Mucuna pruriens y su efecto sobre el rendimiento de maíz dulce (Zea mays L.)
}

\section{Nitrogen contribution to the soil by means of Mucuna pruriens and its effect on the productivity of sweet corn (Zea mays L.)}

\author{
Nitrogen fixation in soil by means of Mucuna pruriens and \\ its effect on the productivity of sweet corn (Zea mays L.)
}

\author{
Oscar Eduardo Sanclemente Reyes ${ }^{1}$, Martín Prager Mosquera² \& Liliana Rocío Beltrán Acevedo³ \\ ${ }^{1}$ Ingeniero ambiental, magíster en ciencias agrarias, Doctor en Agroecología. ${ }^{2}$ Ingeniero Agrónomo, \\ PhD. ${ }^{3}$ Ingeniera ambiental; especialista en administración pública. \\ 1,2 Facultad de Ciencias Agropecuarias, Universidad Nacional de Colombia. AA 237, Palmira, Valle del \\ Cauca, Colombia. ${ }^{3}$ Universidad Nacional Abierta y a Distancia (UNAD); Escuela de Ciencias Agríco- \\ las, Pecuarias y del Medio Ambiente (ECAPMA). Palmira, Colombia. \\ 10sedusancle20@hotmail.com, ${ }^{2}$ pragersa@hotmail.com, ${ }^{3}$ Iilianar.beltran@unad.edu.co
}

\section{Resumen}

Se evaluó el aporte de nitrógeno a un suelo Typic Haplustalfs por parte de la especie Mucuna pruriens como abono verde y cobertura, y su efecto sobre el rendimiento de maíz dulce (Zea mays L.). Se encontró incremento máximo en el suelo de $201 \mathrm{~kg} \mathrm{ha}^{-1}$, la cuál contrastó con el testigo. El tratamiento donde se usó el abono verde más compost $2.5 \mathrm{t} \mathrm{ha}^{-1} \mathrm{y}$ fertilizante de síntesis $125 \mathrm{~kg} \mathrm{ha}^{-1}$, obtuvo el mayor rendimiento de grano seco de maíz $7 \mathrm{t}$ ha-1 el cuál se considera bastante bueno para esta zona agroecológica. El uso de compost y fertilizante de síntesis en la cobertura, no tuvo un efecto significativo sobre el rendimiento del maíz, posiblemente por su dinámica de pérdidas.

Palabras clave: abono verde, cobertura vegetal, fijación de nitrógeno, rendimiento de maíz.

\section{Abstract}

The nitrogen contribution to the soil Typic Haplustalfs through the species Mucuna pruriens as a green manure and cover crop, and its effects on sweet corn yield were evaluated. There was a max fixation in the soil of $201 \mathrm{~kg} \mathrm{ha}^{-1}$ which contrasted with the control. The treatment using the green manure and compost $2.5 \mathrm{t} \mathrm{ha}^{-1}$ and chemical fertilizer $125 \mathrm{~kg} \mathrm{ha}^{-1}$ obtained the highest yield of dry corn grain $7 \mathrm{t} \mathrm{ha}^{-1}$ which is considered very high for this agro ecological zone. The use of compost and fertilizer synthesis as a cover had no significant effect on the corn yield, possibly due to its dynamic losses.

Key-words: green manure, cover crop, nitrogen fixation, corn yield. 


\section{Resumo}

Avaliou-se a entrada de nitrogênio para um solo tipo Typic Haplustalfs pela espécie Mucuna pruriens como adubação verde e cobertura, e seu efeito sob o rendimento de milho doce (Zea mays L.). Encontro-se uma fixação máxima ao solo de $201 \mathrm{~kg}$ ha a qual foi comparada com o controle. $\mathrm{O}$ tratamento onde se usou adubação verde mais de 2,5 t ha ${ }^{-1}$ de composto e fertilizante de síntese 125 $\mathrm{kg}$ foi utilizada ha-1, obteve o maior rendimento de grãos de milho seco $7 \mathrm{t} \mathrm{ha}^{-1}$, o que é considerado bom o suficiente para esta zona agroecológica. O uso de adubo e fertilizantes de síntese na cobertura, não teve efeito significativo sobre a produção de milho, possivelmente devido a suas perdas dinâmicas.

Palavras-chave: adubação verde, cobertura vegetal, fixação de nitrogênio, rendimento de milho.

\section{Introducción}

En la actualidad el uso de abonos verdes y sistemas de cobertura vegetal, se convierten en una alternativa tecnológica para el manejo de cultivos, así como un recurso importante para la conservación de la materia orgánica del suelo (Costa et al., 1992).

La especie Mucuna pruriens ha sido muy empleada en Asia, África y Centroamérica, como abono verde y cultivo de cobertura; tanto en estudios de investigación como en sistemas productivos, gracias a sus distintos efectos sobre algunas propiedades físicas, químicas y biológicas del suelo y el rendimiento en cereales (CIDICCO, 2003).

Uno de los mayores beneficios obtenidos con $\mathrm{Mu}$ cuna pruriens, es la fijación del nitrógeno atmosférico al suelo, debida a su asociación simbiótica con rizobios del suelo. Se han encontrado valores de fijación de hasta $250 \mathrm{~kg} \mathrm{ha}^{-1}$ con ésta especie (Ojiem et al., 2007), lo que beneficia sustancialmente a los cultivos.

El uso de Mucuna pruriens como abono verde y/o cobertura vegetal, involucra el aporte de biomasa rica en nitrógeno orgánico, la cuál sufre un proceso de mineralización y posterior liberación de nutrientes al suelo. El proceso de mineralización del nitrógeno orgánico en el suelo durante el crecimiento de los cultivos, es de importancia ya que puede contribuir en gran parte a la nutrición de los mismos (Dahnke \& Johnson, 1990). La estima- ción de la fracción del nitrógeno mineralizable es compleja ya que es afectada por diversos factores que tienen efecto sobre su dinámica, y están relacionados con los procesos de inmovilización y mineralización, que alteran la disponibilidad del nutriente para el cultivo (Loiseau et al., 1994).

El objetivo de este estudio fue evaluar los cambios en el contenido de nitrógeno de un suelo cultivado con maíz (Zea mays L.) manejado con un abono verde y una cobertura de Mucuna pruriens.

\section{Materiales y métodos}

Este estudio se desarrolló en un suelo de la cordiIlera central, ubicado en el municipio de Palmira, Valle del Cauca, Colombia. El sitio del ensayo de estableció a los 1350 m.s.n.m., el cuál presenta una temperatura media anual de $23^{\circ} \mathrm{C}$ y un $70 \%$ de húmedad relativa del aire. El uso del suelo antes de ensayo fue un barbecho enriquecido biológicamente por 10 años, el cuál se intervino para establecer las parcelas experimentales.

La clasificación taxonómica del suelo es Typic Haplustalfs (IGAC, CVC; 2004), presenta una textura Franco Arenosa (FA) dentro de sus primeros 10 $\mathrm{cm}$ de profundidad, con un porcentaje de arena del $61.6 \% ; 15.7 \%$ de arcilla y $22.7 \%$ de limo. Los resultados de los análisis químicos iniciales fue- 
ron los siguientes: $\mathrm{pH}\left(\mathrm{H}_{2} \mathrm{O}\right)=6.0$, contenido de nitrógeno total $\left(\mathrm{g} \mathrm{kg}^{-1}\right)=1.6$; contenido de nitrógeno inorgánico $\left(\mathrm{NO}_{3}+\mathrm{NH}_{4}\right)$ en $\left(\mathrm{mg} \mathrm{kg}^{-1}\right)=35.0$; bases cambiables $\left(\mathrm{cmol} \mathrm{kg}^{-1}\right)$ : $\mathrm{Ca}=26.1 ; \mathrm{K}=0.3 ; \mathrm{Al}=0.1$; $\mathrm{Mg}=23.3$ y $\mathrm{P}(\mathrm{ppm})=3.5$.

Se utilizó un diseño experimental en bloques completos al azar (BCAA) con tres repeticiones. El tamaño de la parcela experimental fue de 20 $\mathrm{m}^{2}$. Se asignaron 7 tratamientos a las unidades experimentales en cada bloque, para un total de 21 parcelas con un área de $420 \mathrm{~m}^{2}$.

Los tratamientos asignados fueron: T1: Testigo (Sin Mucuna pruriens y sin fertilizar), T2: AVSF (Abono verde sin fertilizar), T3: AVO (Abono verde más abono orgánico compostado), T4: AVQ (Abono verde más fertilizante de síntesis química), T5: AVOQ (Abono verde más abono orgánico compostado complementado con fertilizante de síntesis química), T6: CMSF (Cobertura muerta sin fertilizar), T7: CMOQ (Cobertura muerta más abono orgánico compostado complementado con fertilizante de síntesis química).

El establecimiento del cultivo de Mucuna pruriens en las correspondientes parcelas experimentales, se realizó utilizando $70 \mathrm{~kg} \mathrm{ha}^{-1}$ de semilla; posteriormente se incorporó in situ la biomasa vegetal como abono verde y/o como cobertura muerta, a los 80 d.d.s. (días después de sembrado).

La incorporación del abono verde se realizó con machete, a una profundidad de $5 \mathrm{~cm}$; evitando disturbar el suelo. La cobertura muerta se obtuvo después de la aplicación del herbicida glifosato (N-fosfonometilglicina), en dosis comercial.

Después de 20 días de establecida la cobertura muerta e incorporar el abono verde, se realizó la siembra del cultivo de maíz (Zea mays L.) variedad ICA 305. La densidad de siembra utilizada fue 40.000 plantas ha ${ }^{-1}$.

El abonamiento se realizó en dos etapas del cultivo de maíz: a los 10 d.d.s y a los 30 d.d.s., fraccionando las dosis en $50 \%$ para cada aplicación. El abono orgánico utilizado fue compost tipo bocachi en dosis de $5 \mathrm{t} \mathrm{ha}^{-1}$. El fertilizante de síntesis química utilizado fue NPK 10-30-10 se aplicó al tratamiento en dosis de $250 \mathrm{~kg} \mathrm{ha}^{-1}$. En los tratamientos T5 y T7, se utilizó una enmienda conformada por $2.5 \mathrm{t} \mathrm{ha}^{-1}$ de compost tipo bocachi complementada con $125 \mathrm{~kg} \mathrm{ha}^{-1}$ del fertilizante de síntesis química NPK 10-30-10.

La determinación del nitrógeno total se realizó por espectrometría mediante método Kjeldahl citado por (Radojevic et al., 2002); donde las muestras de suelo se digestan con $\mathrm{H}_{2} \mathrm{SO}_{4}$ más la adición de un catalizador compuesto de $\mathrm{Cu}, \mathrm{Se}$ y $\mathrm{KSO}_{4}$. La determinación del $\mathrm{N}$ se realizó en un analizador de nutrientes (espectrofotómetro UV- VIS). $\mathrm{NO}_{3}$ y $\mathrm{NH}_{4}$, se determinaron por extracción con $\mathrm{KCl} 1 \mathrm{M}$ y posterior lectura en espectrofotómetro.

El Nitrógeno Mineralizable, se estimó mediante el método de incubación anaeróbica descrito por (Gianello \& Bremner, 1986) donde se determina la cantidad de $\mathrm{NH}_{4}$ por espectrometría antes y después, de una incubación anaeróbica de $20 \mathrm{~g}$ de suelo con contenido de humedad cercano a capacidad de campo, en tubos de ensayo sellados y encubados a una temperatura de $37^{\circ} \mathrm{C}$, durante 14 días; siendo el diferencial del contenido de $\mathrm{NH}_{4}$ antes y después de la incubación, el $\mathrm{N}$ que pasó desde su estado orgánico a inorgánico por acción microbiana. $\mathrm{N}$ mineralizable $\left(\mathrm{mg} \mathrm{kg}^{-1}\right)=\mathrm{NH}_{4}$ antes de incubar - $\mathrm{NH}_{4}$ después de incubación.

El aporte de Nitrógeno efectivo, se estimó como la diferencia entre el contenido de nitrógeno inorgánico $\left(\mathrm{kg} \mathrm{ha}^{-1}\right)$ en las parcelas con Mucuna pruriens y el testigo, tomando una capa de suelo de $10 \mathrm{~cm}$. $N$ fijado $\left(\mathrm{kg} \mathrm{ha}^{-1}\right)=N$ suelo con Mucuna pruriens $-N$ suelo testigo.

Los resultados obtenidos en las pruebas de laboratorio, se trataron estadísticamente usando el programa SAS versión 9.0; de ANOVA $(P<0.1)$ y prueba de comparación de medias con Duncan $(P<0.1)$. 


\section{Resultados y discusión}

La Figura 1 presenta el contenido de nitrógeno total del suelo. El establecimiento de la Mucuna pruriens logró incrementar los valores de nitrógeno total del suelo entre $31 \%$ y $71 \%$ en comparación con el testigo que tuvo pérdidas significativas $(\mathrm{P}<0.1)$, mostrando los beneficios de esta especie como aportadora de nitrógeno. La utilización del compost en el abono verde y la cobertura, permiten hacer un uso más eficiente del nitrógeno del suelo, lo que se refleja en la obtención de excedentes al final de la cosecha de maíz.

El contenido de nitrógeno inorgánico (NO3+NH4), se presenta en la Figura 2. El uso de la cobertura muerta de Mucuna pruriens permitió un aporte significativamente alto $(P<0.1)$ de nitrógeno a la plantación de maíz, en comparación con el testigo. La utilización del abono verde de Mucuna pruriens logró un menor aporte de nitrógeno inorgánico al cultivo de maíz, en comparación con la cobertura muerta. Es posible que la cobertura muerta de Mucuna pruriens no haya retenido el nitrógeno inorgánico suministrado mediante la fertilización, y en cambio este componente se perdió mediante procesos de lixiviación y/o lavado, gracias a su alta movilidad.

El aporte efectivo del nitrógeno al suelo, estuvo entre 51 y $237 \mathrm{~kg} \mathrm{ha-}{ }^{1}$; siendo bastante alto si consideramos que los requerimientos para la variedad de maíz (Zea mays L.) ICA 305 son aproximadamente de $100 \mathrm{~kg} \mathrm{ha}-{ }^{1}$ lo que permite hacer un uso más eficiente de los fertilizantes de síntesis química.
La Figura 3, presenta el contenido de nitrógeno mineralizable del suelo. La tasa más baja de mineralización del nitrógeno orgánico en la primera etapa de muestreo, se presentó en las parcelas donde se estableció el tratamiento testigo con un valor de $61 \mathrm{mg} \mathrm{kg}^{-1}$ de suelo. Estos valores se deben a que el uso de Mucuna pruriens como abono verde o cobertura muerta, aporta una cantidad considerable de nitrógeno orgánico presente en su biomasa en formas proteicas, de aminoácidos y ácidos nucléicos; los cuáles pasan a ser $\mathrm{NH} 4$ en el suelo mediante acción microbiana, como inicio del proceso de mineralización del nitrógeno.

Al final del la etapa de cosecha de maíz (Zea mays L.), el tratamiento donde se usó el abono verde más compost complementado con fertilizante de síntesis, presentó el valor más alto de nitrógeno mineralizable de $43 \mathrm{mg} \mathrm{kg}^{-1}$ lo que indica un remanente importante para la fertilización del próximo cultivo.

La Figura 4 presenta los resultados del rendimiento en grano seco de maíz (Zea mays L.). La mayor productividad $7 \mathrm{t} \mathrm{ha-}{ }^{-1}$ se obtuvo con el uso de $\mathrm{Mu}$ cuna pruriens como abono verde complementado con el compost y fertilizante de síntesis, este valor fue significativamente mayor $(\mathrm{P}<0.1)$ que el testigo. El uso de cobertura muerta complementado con el compost y fertilizante de síntesis no difirió en rendimiento con el tratamiento donde se usó la cobertura muerta sin fertilizar, lo que podría indicar posibles pérdidas del fertilizante por acción de lavado y/o lixiviación, gracias a su alta movilidad. La decisión de aplicar fertilizante dependerá de los precios de éste versus los del cultivo de maíz. 


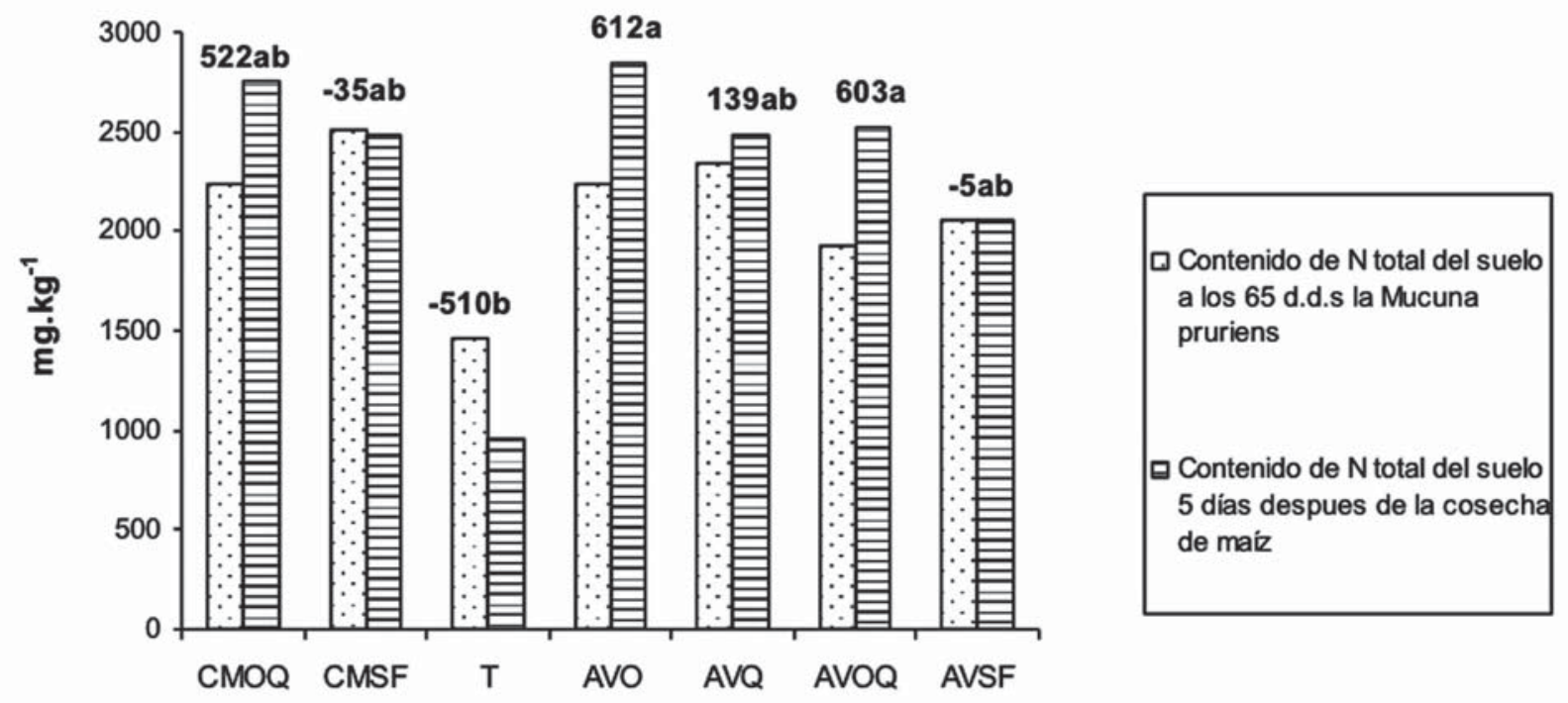

Figura 1. Contenido de nitrógeno total del suelo de 0-10 cm, en $\left(\mathrm{mg}^{\mathrm{kg}} \mathrm{kg}^{-1}\right)$, durante dos etapas del experimento: A los 65 d.d.s la Mucuna pruriens y 5 días después de la cosecha del maíz. Los valores en las barras indican la diferencia entre los dos periodos, se usó una prueba de Duncan $(P<0.1)$.

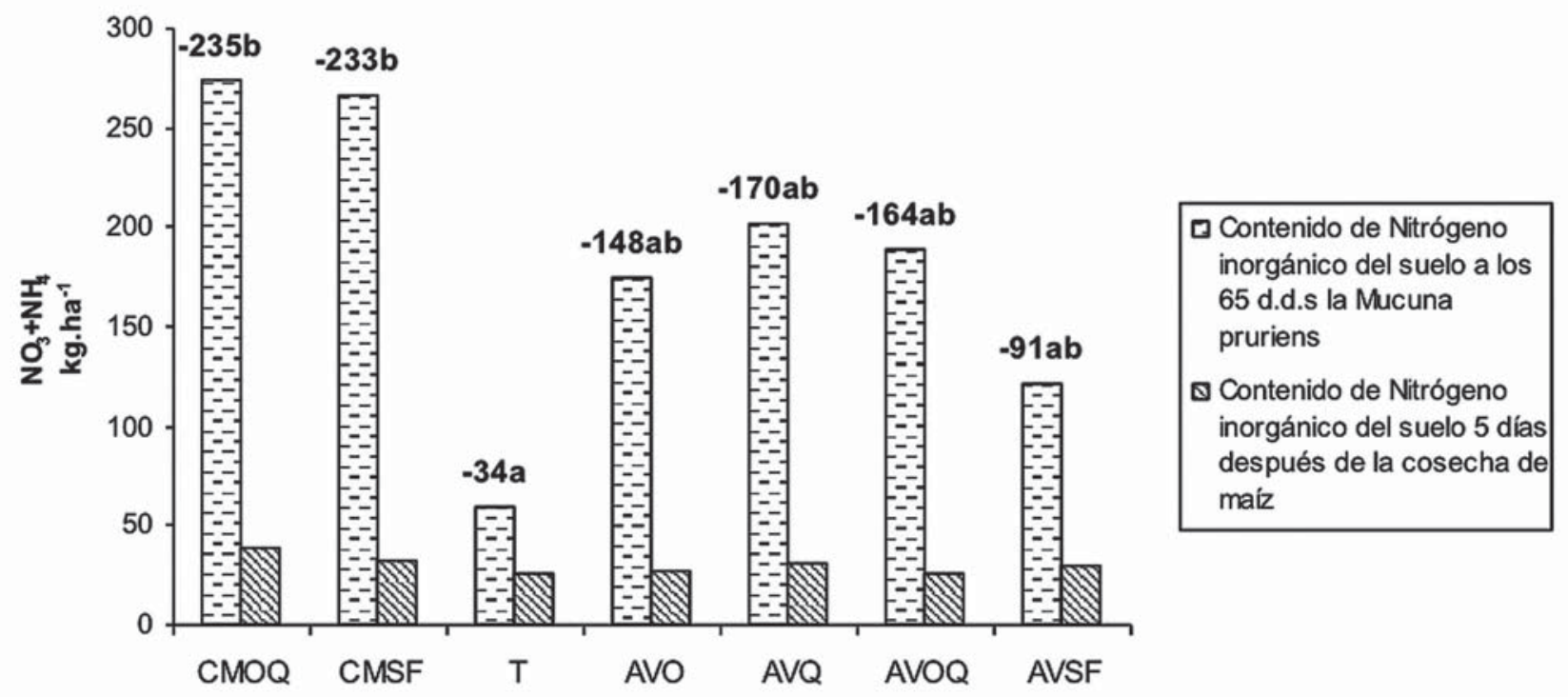

Figura 2. Contenido de Nitrógeno inorgánico $\left(\mathrm{NO}_{3}+\mathrm{NH}_{4}\right)$ del suelo de 0-10 $\mathrm{cm}$ en $\left(\mathrm{kg} \cdot \mathrm{ha}^{-1}\right)$, durante dos etapas del ensayo: a los 65 d.d.s la Mucuna pruriens y 5 días después de la cosecha de maíz. Los valores en las barras muestran la diferencia entre los dos periodos de muestreo. Se usó una prueba de Duncan $(P<0.1)$. 


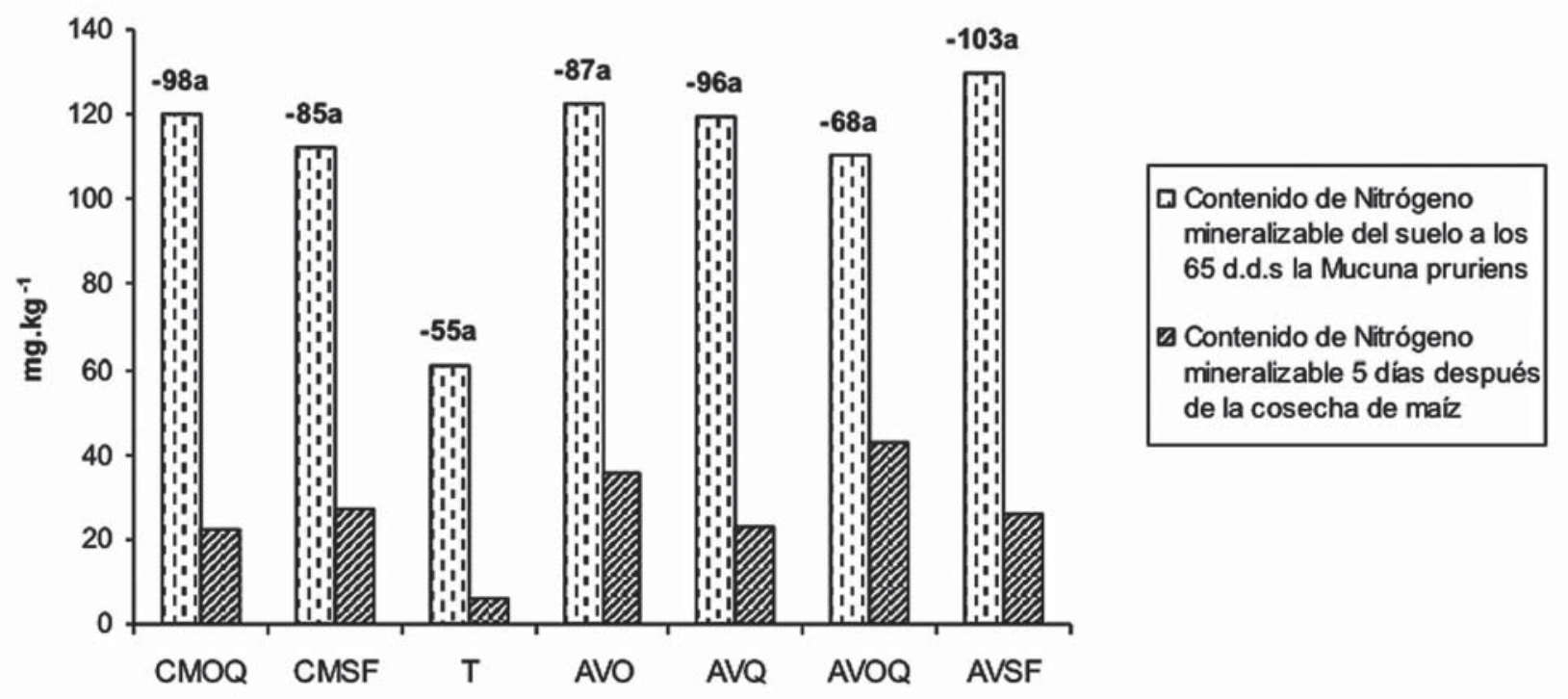

Figura 3. Contenidos de Nitrógeno mineralizable del suelo de $0-10 \mathrm{~cm}$, en $\left(\mathrm{mg}^{\mathrm{kg}} \mathrm{kg}^{-1}\right)$, durante dos etapas del experimento: A los 65 d.d.s la Mucuna pruriens

y 5 días después de la cosecha del maíz. Los valores en las barras muestran la diferencia entre los dos periodos de muestreo. Se usó una prueba de Duncan $(\mathrm{P}<0.1)$.

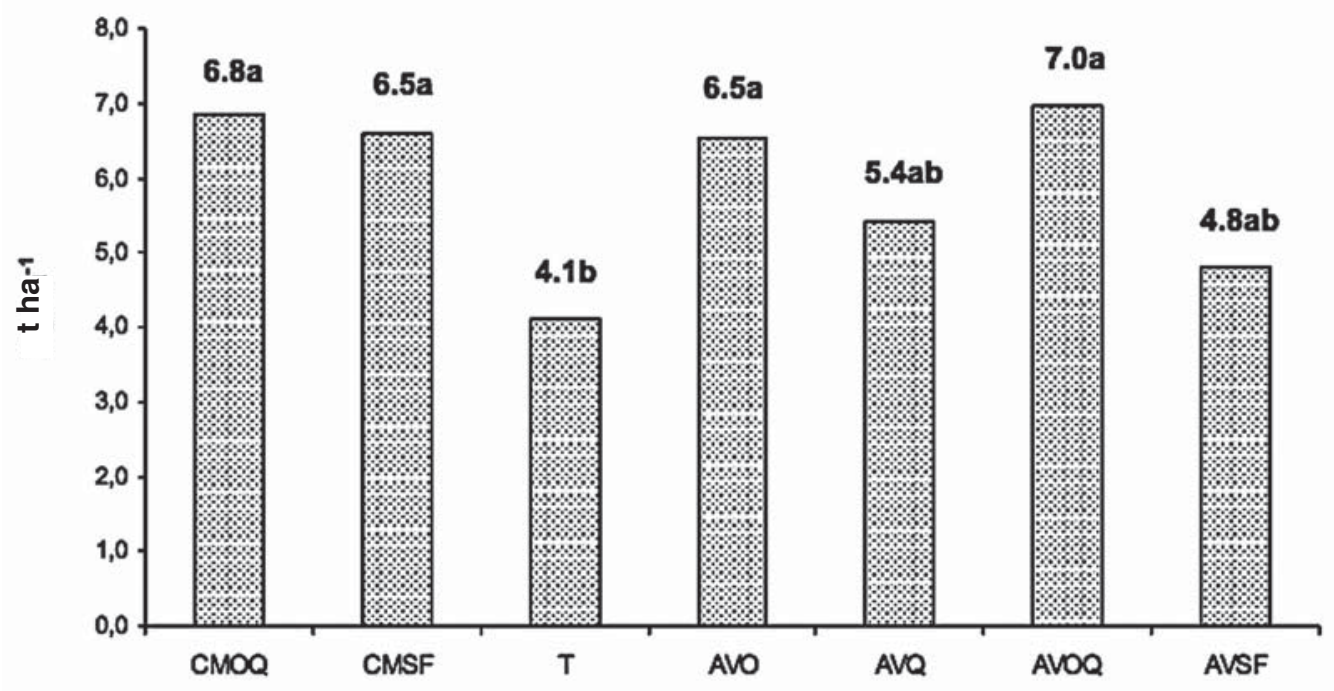

Figura 4. Rendimiento de grano seco de maíz en t.ha-1. Se usó una prueba de Duncan $(P<0.1)$. 


\section{Conclusiones}

La utilización de la especie Mucuna pruriens, como alternativa tecnológica en el cultivo de maíz, permite hacer un uso más eficiente del nitrógeno en el suelo, factor que está en dirección con el proceso de sostenibilidad del recurso. La asociación simbiótica de Mucuna pruriens con los rizobios del suelo, posibilitó el ciclaje del nitrógeno atmosférico al suelo de hasta $201 \mathrm{~kg}$ ha- ${ }^{1}$ beneficiando de esta forma al cultivo de maíz (Zea mays L.) que alcanzó un rendimiento máximo de $7 \mathrm{t} \mathrm{ha}^{-1}$. La incorporación de Mucuna pruriens como abono verde, incrementó los procesos de mineralización del nitrógeno en el suelo gracias al aporte considerable de éste nutriente con su biomasa, factor que permitió obtener remanentes de nitrógeno asimilable en el suelo para próximos cultivos.

\section{Literatura citada}

1. CENTRO INTERNACIONAL DE INFORMACIÓN SOBRE CULTIVOS DE COBERTURA. CIDICCO. (2003). Catalogo de Abonos verdes / cultivos de cobertura (CCAV), empleados por pequeños productores de los trópicos. Honduras.

2. Costa, M.; Calegari, A.; Mondardo, A.; Bulisani, E.A.; Wildner, L. Do P.; Alcântara, P.B.; Miyasaka, S. \& Amado, T.J.C. (1992). Adubação verde no sul do Brasil. Rio de Janeiro, ASPTA. $346 \mathrm{p}$.

3. Dahnke, J.. (1990). Testing soils for available nitrogen. En: Westerman RL et al. (Eds.). Soil Testing and Plant Analysis. No 3 Soil Science Society of America, Madison Wisconsin, EEUU. 127-139 pp.

4. Gianello, C. \& Bremner, J. (1986). A simple chemical method of assessing potentially vailable organic nitrogen in soil. Commun. Soil Sci. Plant Anal.No 17. 195-214 pp.

5. Instituto Geográfico Agustín Codazzi IGAC \& Corporación Autónoma Regional del Valle del Cauca CVC. (2004). Levantamiento de suelos y zonificación de tierras del Departamento de valle del Cauca 2004.

6. Loiseau, P., Chaussod, R., Delpy, R. (1994). Soil microbial biomass and in situ nitrogen mineralization after 20 years of different nitrogen fertilization and forage cropping systems. European J. Agron., 3:327-332.

7. Ojiem, J., Vanlauwe, B., Ridder, N. \& Giller, K. (2007). Niche-based assessment of contributions of legumes to the nitrogen economy of Western Kenya smallholder farms. Journal Plant soil No 292: 119-135.

8. Radojevic, M. \& Bashkin, V. (2002). Practical Environmental Analysis. RSC Publishing. 